\title{
Dexmedetomidine Improves Locomotor Function and Alleviates Thermal Hyperalgesia Following Sciatic Nerve Crush Injury in
} Rats

\author{
Myung-Soo Jang ${ }^{1}$, Jin Hee Han¹, Dong-Ok Kim², Gil Woo² , Jae-Ik Kwon², Jun-Young Chung ${ }^{2}$ Bong-Jae Lee², Jae-Woo Yi \\ ${ }^{1}$ Department of Anesthesiology and Pain Medicine, Kyung Hee University Medical Center, College of Medicine, Kyung Hee University, Seoul, Korea \\ ${ }^{2}$ Department of Anesthesiology and Pain Medicine, Kyung Hee University Hospital at Gangdong, College of Medicine, Kyung Hee University, Seoul, Korea
}

Purpose: The effects of dexmedetomidine on locomotor function and thermal hyperalgesia in sciatic nerve crush injury (SNCI) were investigated using rats.

Methods: After exposing the right sciatic nerve, the sciatic nerve was crushed for 1 minute by a surgical clip. One day after nerve injury, dexmedetomidine $(5,25$, and $50 \mu \mathrm{g} / \mathrm{kg}$ ) was directly applied to the injured sciatic nerve once a day for 14 days. Walking track analysis was used to assess locomotor function and plantar test was conducted to assess thermal pain sensitivity. Immunohistochemistry was performed to determine the expression of c-Fos in the ventrolateral periaqueductal gray (vlPAG) and paraventricular nucleus (PVN). Western blot was used to evaluate the expression level of nerve growth factor (NGF) and myelin basic protein (MBP) in the sciatic nerve.

Results: SNCI resulted in deterioration of locomotor function and increased thermal pain sensitivity. The level of c-Fos expression in the PVN and vlPAG was increased and the level of NGF and MBP expression in the sciatic nerve was enhanced by SNCI. Dexmedetomidine treatment improved locomotor function and upregulated expression of NGF and MBP in the sciatic nerve of SNCI. Dexmedetomidine treatment alleviated thermal hyperalgesia and downregulated expression of c-Fos in the vlPAG and PVN after SNCI.

Conclusions: Dexmedetomidine may be used as a potential new treatment drug for recovery of locomotion and control of pain in peripheral nerve injury.

Keywords: Dexmedetomidine; Locomotion; Pain; Peripheral nerve injury; Sciatic nerve

- Fund/Grant Support: This work was supported by a grant from Kyung Hee University in 2015 (grant number: KHU-20151261).

- Research Ethics: The experimental design was approved by the Institutional Care and Use Committee of Kyung Hee University

(KHUASP[SE]-17-094)

- Conflict of Interest: No potential conflict of interest relevant to this article was reported.

\footnotetext{
- HIGHLIGHTS

- Sciatic nerve crush injury resulted in deterioration of locomotor function and increased thermal pain sensitivity.

- Dexmedetomidine treatment improved locomotor function.

- Dexmedetomidine treatment alleviated thermal hyperalgesia.
}

Corresponding author: Jae-Woo Yi (iD https://orcid.org/0000-0001-6474-5624 Department of Anesthesiology and Pain Medicine, Kangdong Kyung Hee Hospital, College of Medicine, Kyung Hee University, 892 Dongnam-ro, Gangdong-gu, Seoul 05278, Korea

E-mail: mdyjwchk@khu.ac.kr / Tel: +82-2-440-6191 / Fax: +82-2-440-7808

Submitted: April 2, 2020 / Accepted after revision: May 5, 2020 


\section{INTRODUCTION}

Peripheral nerve injury is usually encountered in clinical practice and often causes deficits in motor function [1]. Damage to the peripheral nervous system causes secondary complication such as permanent impairment of sensorimotor function and neuropathic pain, leading to loss of function and deteriorating quality of life [2]. Sciatic nerve crush injury (SNCI) is a type of painful neuropathy, and the affected limb expresses hyperalgesia, abnormal gait, and swelling [3].

Neuropathic pain is clinically classified as irritation-independent pain (or spontaneous progressive pain) and irritation-dependent pain (or induced pain). Induced pain depends on the presence of a stimulus, and it is characterized by hyperexcitability of the nervous system [4]. The afferent fibers in periaqueductal gray (PAG) and paraventricular nucleus (PVN) process nociceptor by receiving signals from nociceptive neurons of the spinal cord and sending projection to the thalamic nuclei. Expression of c-Fos can be used as an indicator of neuronal activation according to noxious stimulation [5]. c-Fos is one of the immediate early genes, and expression of c-Fos is altered by the metabolic activity of neurons [6]. Expression of c-Fos is also used to assess neuronal responses to various internal stimuli [7].

Peripheral nerve damage causes many reactions in the cells surrounding the injury site and affects the progression of axonal degeneration and regeneration occurring early in the injury [8]. Proper control of cell signaling and gene expression is important in regulating degenerative and regenerative potentials [9].

Animal experiments and clinical studies have identified that neurotrophic factors are important in enhancing nerve regeneration $[10,11]$. Among neurotrophic factors, nerve growth factor (NGF) is implicated in the regulation of survival, growth, and differentiation of neurons [12]. Topically applied NGF stimulated nerve regeneration and promoted functional recovery in SNCI rats [13]. Another important factor for peripheral nerve regeneration is myelin basic protein $(\mathrm{MBP})$, which is also affected by SNCI $[9,14]$. MBP is expressed in oligodendrocytes and Schwann cells, and MBP has a regulatory function in myelination [15].

Dexmedetomidine, selective $\alpha_{2}$-adrenoreceptor agonist, acts as an analgesic, sedative, and anesthetic [16]. Moreover, dexmedetomidine is approximately 8 times more selective for the $\alpha_{2}$ adrenoceptor than clonidine [17]. Clinically, dexmedetomidine has shown desirable analgesic property against acute inflamma- tory pain, postoperative pain, and even neuropathic pain unresponsive to opioid analgesics [18,19]. Clinical effects of dexmedetomidine on various types of pain are well-established; however, the efficacy of dexmedetomidine for sciatic nerve regeneration after SNCI was not proved.

In this experiment, the effect of dexmedetomidine on locomotor function and thermal hyperalgesia following SNCI was verified using rats. Walking tract analysis and plantar test were performed. Immunohistochemistry was used to determine cFos expression in PVN and vlPAG. NGF and MBP expression in the sciatic nerve was calculated by western blot.

\section{MATERIALS AND METHODS}

\section{Animals and Treatments}

Male Sprague-Dawley rats weighing $250 \pm 10$ g (12 weeks old) were used for this experiment. We followed the Guidelines for the Care and Use of Animals approved by the National Institute of Health Council for the management and use of laboratory animals. This experimental design was approved by the Institutional Care and Use Committee of Kyung Hee University (KHUASP[SE]-17-094). The animals were randomly classified into the following 5 groups ( $\mathrm{n}=8$ in each group): sham-operated group, SNCI-induced group, SNCI-induced and $5-\mu \mathrm{g} / \mathrm{kg}$ dexmedetomidine treatment group, SNCI-induced and $25-\mu \mathrm{g} / \mathrm{kg}$ dexmedetomidine treatment group, and SNCI-induced and 50$\mu \mathrm{g} / \mathrm{kg}$ dexmedetomidine treatment group. For the dexmedetomidine treatment groups, the appropriate dosage of dexmedetomidine (Precedex, Pfizer, NY, USA) was injected directly into the injury site $(200 \mu \mathrm{L})$ one time per a day during 10 days, starting 3 days after SNCI induction. Previous study was considered for determining the dosage of dexmedetomidine [20]. The rats in the sham-operated group and in the SNCI-induced group received direct injection of $200 \mu \mathrm{L} 0.9 \% \mathrm{NaCl}$ into the right sciatic nerve for the same duration.

\section{Induction of Sciatic Nerve Crushed Injury}

SNCI was induced by the following experimental protocol [5]. The rats were anesthetized with Zoletil $50(40 \mathrm{mg} / \mathrm{kg}$; Virbac Laboratories, Carros, France). After exposing the right sciatic nerve, the sciatic nerve was crushed for 1 minute with a surgical clip at a pressure $125 \mathrm{~g}$ (Fine Science Tools Inc., San Francisco, CA, USA). For the sham-operated group, the sciatic nerve was exposed, but the nerve was not crushed. 


\section{Walking Tract Analysis}

To assess locomotor recovery, walking track analysis was carried out as previous described method [5]. Walking track analysis was performed $0,2,8$, and 11 days following SNCI. First, the rats were placed on an $8 \times 66-\mathrm{cm}$ walking track with a sheet of white paper at the bottom of the track. When the rats walked, red ink was applied to the hind paw to leave fingerprints on the paper. The maximum value of each measurement was used for the analysis, and the print length (PL), toe spread (TS), and middle toe spread (IT) were all measured. Data were recorded with the prefix $\mathrm{E}$ for the experimental side and $\mathrm{N}$ for the nonoperated side. From the intact left (nonoperated) foot (NPL, NTS, and NIT) and the injured right (experimental) foot (EPL, ETS, and EIT), these parameters were obtained. The sciatic functional index (SFI) is an indicator of the degree of nerve dysfunction and was calculated using a formula previously reported [5]. The following formula was used for data acquisition: $\mathrm{SFI}=(-38.3 \pm \mathrm{PLF})+(109.5 \pm \mathrm{TSF})+(13.3 \pm \mathrm{ITF})-8.8$. Print length factor $(\mathrm{PLF})=(\mathrm{EPL}-\mathrm{NPL}) / \mathrm{NPL}$. Toe spread factor $(\mathrm{TSF})=($ ETS-NTS $) /$ NTS. Intermediary toe spread factor $(\mathrm{ITF})=($ EIT-NIT $) /$ NIT. SFI varies from 0 to $-100,0$ corresponds to normal function, and -100 corresponds complete dysfunction.

\section{Plantar Test}

Plantar test was performed using an algesimeter (Ugo-Basile, Comerio, Italy). Thermal hyperalgesia was determined 12 days after SNCI. The rats were positioned in a plastic box and acclimatized to the testing space for at least 5 minutes before starting the plantar test. After acclimatization, radiant heat was applied to the plantar surface of the ipsilateral hind paw until the rat pull out. When the reflected light was blocked (for example, when the animal lifted its feet), the photocell automatically turned off the heat source. The time was recorded as the withdrawal latency of the paw (seconds). In order to induce activation of nonmyelinated fibers, the strength was set to a low power $\left(40 \mathrm{~mW} / \mathrm{cm}^{2}\right)$ at a heating rate of $1^{\circ} \mathrm{C} / \mathrm{sec}$.

\section{Tissue Preparation}

According to the previous described method [16,21], tissue preparation was performed. Immediately after undergoing the plantar test, the animals were sacrificed. Zoletil $50(40 \mathrm{mg} / \mathrm{kg}$, intraperitoneally; Vibac Laboratories) was used to anesthetize the rats, and the crushed sciatic nerve was removed. And then, $50 \mathrm{mM}$ phosphate-buffered saline (PBS) was transcardially per- fused with a freshly prepared solution consisting with $4 \%$ paraformaldehyde in $100 \mathrm{mM}$ phosphate buffer $(\mathrm{pH}, 7.4)$. Brains were dissected and then stored overnight in the same paraformaldehyde solution. For cryoprotection, the brains were immersed in 30\% sucrose. Using a cryostat (Leica, Nussloch, Germany), 40- $\mu \mathrm{m}$ thick coronal sections were made. Ten sections on average containing PVN and vlPAG were collected.

\section{Immunohistochemistry for c-Fos}

According to the previous described method [3,22], we conducted c-Fos immunohistochemistry to detect c-Fos expression in the PVN and vlPAG. Rabbit anti-c-Fos antibody (1:1,000; Santa Cruz Biotechnology, Santa Cruz, CA, USA) was incubated with the free-floating sections overnight. Biotinylated antirabbit secondary antibody (1:200; Vector Laboratories, Burlingame, CA, USA) was incubated with the sections for 1 hour. Then, avidin-biotin-peroxidase complex (Vector Laboratories) was incubated with the sections at room temperature during 1 hour. The sections were incubated with a solution containing $0.03 \%$ 3,3-diaminobenzidine and $0.01 \% \mathrm{H}_{2} \mathrm{O}_{2}$ in $50 \mathrm{mM}$ Trisbuffer ( $\mathrm{pH}, 7.6)$ for 3 minutes, and immunoreactivity was visualized. The sections were mounted on gelatin-coated slides after washing 3 times with PBS. The slides were covered with coverslips by Permount (Fisher Scientific, New Jersey, NJ, USA), followed air dry at room temperature overnight.

\section{Western Blotting for NGF and MBP}

According to the previous described method [16], we conducted western blot analysis to detect NGF and MBP expression in the sciatic nerve. Sciatic nerve tissues were homogenized on ice and then lysed in lysis buffer. Using a Bio-Rad Colorimetric Protein Assay kit (Hercules, CA, USA), the amount of protein in each sample was quantitated. By sodium dodecyl sulfatepolyacrylamide gel, protein $(30 \mu \mathrm{g})$ was separated, and then the protein was transferred onto a nitrocellulose membrane. The membrane was incubated in 5\% skim milk of Tris-buffered saline with $0.1 \%$ Tween-20. The membrane was incubated with rabbit anti-NGF antibody and goat anti-MBP antibody (1:1,000; Santa Cruz Biotechnology) overnight at $4^{\circ} \mathrm{C}$. The membrane was subsequently incubated with secondary antibody $(1: 2,000$; Vector Laboratories) during 1 hour. For band detection, an enhanced chemiluminescence detection kit (Santa Cruz Biotechnology) was used, and analyzed the detected band using Molecular Analyst (version 1.4.1, Bio-Rad). 


\section{Data Analysis}

Immunoreactive bands of NGF and MBP were analyzed by an Image-Pro Plus imaging system (Media Cyberbetics Inc., Silver Spring, MD, USA). The number of c-Fos-positive cells in the PVN and vlPAG was calculated hemilaterally through a light microscope (Olympus, Tokyo, Japan). One-way analysis of variance with Duncan post hoc test was used for statistical analysis. The experiment results were shown as the mean \pm standard error of the mean and $\mathrm{P}<0.05$ was given significance.

\section{RESULTS}

\section{Effect of Dexmedetomidine on Locomotor Function}

Locomotor function was determined by walking tract analysis (Fig. 1). SFI was measured on days 2, 8, and 11 after SNCI. SFI in the sham-operated group remained constant during the experiment for approximately -10 to -20 . At the beginning of the experiment, SFI in all SNCI groups had fallen to near -100. In the SNCI groups, the SFI slowly changed during the experiment. However, in the dexmedetomidine treatment groups, the increase of SFI appeared on day 8 after induction of SNCI $(\mathrm{P}<0.05)$. On day 11 after SNCI induction, the $50-\mu \mathrm{g} / \mathrm{kg}$ dexmedetomidine treatment group showed more palpation of re-

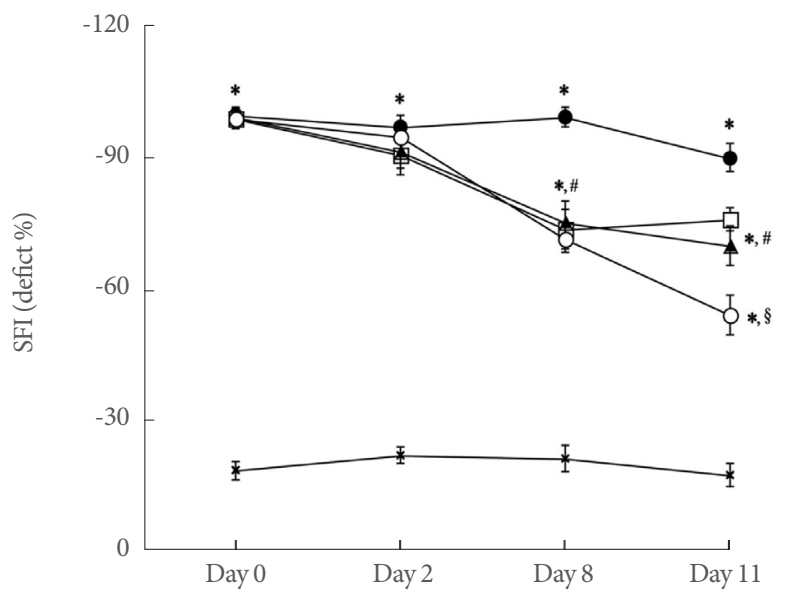

Fig. 1. Effect of dexmedetomidine treatment on sciatic functional index (SFI) following sciatic nerve crush injury. $\mathrm{x}$, shamoperated group; $\bullet$, sciatic nerve crush injury (SNCI)-induced group; $\square$, SNCI-induced and $5-\mu \mathrm{g} / \mathrm{kg}$ dexmedetomidine treatment group; $\boldsymbol{\Lambda}, \mathrm{SNCI}-$ induced and $25-\mu \mathrm{g} / \mathrm{kg}$ dexmedetomidine treatment group; $\circ$, SNCI-induced and $50-\mu \mathrm{g} / \mathrm{kg}$ dexmedetomidine treatment group. ${ }^{\star} \mathrm{P}<0.05$ compared to the sham-operation group. ${ }^{*} \mathrm{P}<0.05$ compared to the SNCI-induced group. ${ }^{\circledR} \mathrm{P}<0.05$ compared to the SNCI-induced and $5-\mu \mathrm{g} / \mathrm{kg}$ dexmedetomidine treatment group. covery $(\mathrm{P}<0.05)$. The present results suggest that treatment with dexmedetomidine improved locomotor function after SNCI induction.

\section{Effect of Dexmedetomidine on Thermal Pain Sensitivity}

Plantar test was conducted to measure thermal pain sensitivity (Fig. 2). Induction of SNCI significantly decreased the paw withdrawal latency $(\mathrm{P}<0.05)$. In contrast, dexmedetomidine treatment significantly increased paw withdrawal latency according to the dose-dependent manner $(\mathrm{P}<0.05)$. These results indicate that treatment with dexmedetomidine alleviated thermal hyperalgesia following SNCI.

\section{Effect of Dexmedetomidine on c-Fos Expression.}

Fig. 3 represents the relative level of c-Fos expression in the PVN and vlPAG. Induction of SNCI significantly enhanced expression of c-Fos in the PVN and vlPAG $(\mathrm{P}<0.05)$. In contrast, dexmedetomidine treatment significantly inhibited SNCI-induced c-Fos expression according to the dose-dependent manner $(\mathrm{P}<0.05)$. The present results suggest that treatment with dexmedetomidine ameliorated pain transmission caused by SNCI.

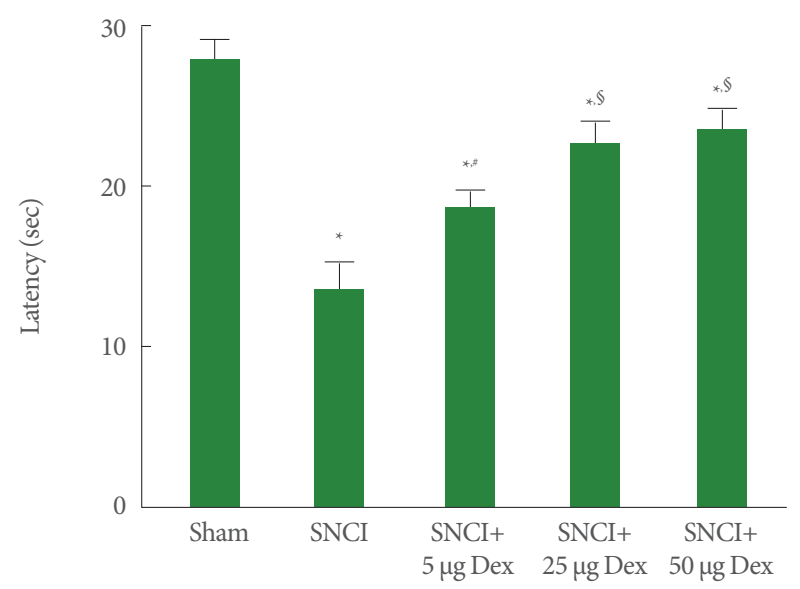

Fig. 2. Effect of dexmedetomidine on thermal hyperalgesia in the plantar test. Sham, sham-operated group; SNCI, sciatic nerve crush injury-induced group; SNCI+5 $\mu$ g Dex, SNCI-induced and $5-\mu \mathrm{g} / \mathrm{kg}$ dexmedetomidine treatment group; SNCI+25 $\mu \mathrm{g}$ Dex, SNCI-induced and $25 \mu \mathrm{g} / \mathrm{kg}$ dexmedetomidine treatment group; SNCI+50 $\mu \mathrm{g}$ Dex, SNCI-induced and 50$\mu \mathrm{g} / \mathrm{kg}$ dexmedetomidine treatment group. ${ }^{\star} \mathrm{P}<0.05$ compared to the sham-operated group. ${ }^{*} \mathrm{P}<0.05$ compared to the SNCIinduced group. ${ }^{5} \mathrm{P}<0.05$ compared to the $\mathrm{SNCI}$-induced and $5-\mu \mathrm{g} / \mathrm{kg}$ dexmedetomidine treatment group. 


\section{Effects of Dexmedetomidine on NGF and MBP Expression}

Fig. 4 represents the relative level of NGF and MBP expression in the sciatic nerve. The level of NGF and MBP expression in the SNCI-induced group was higher than in the sham-operated group $(\mathrm{P}<0.05)$. Moreover, treatment with dexmedetomidine resulted in overexpression of NGF and MBP in the SNCI rats according to the dose-dependent manner $(\mathrm{P}<0.05)$. These results indicate that treatment with dexmedetomidine facilitated SNCI-induced NGF and MBP expression.

\section{DISCUSSION}

Increase of SFI represents improvement of locomotor function and decrease of SFI represents exacerbation of locomotor function. In our study, the rats with SNCI showed a characteristic footprint pattern, indication a decrease in the SFI value. How- ever, the SFI values in the dexmedetomidine treatment groups were sharply increased beginning at 8 days after SNCI. In the SFI values determined by the last walking tract analysis, $50-\mu \mathrm{g} / \mathrm{kg}$ dexmedetomidine treatment showed the strongest increase in SFI value.

In SNCI, the affected limb displays hyperalgesia, abnormal gait due to pain, and swelling [23]. Sciatic nerve damage is known to cause cold allodynia and heat hyperalgesia [23]. The plantar test has been used to evaluate heat hypersensitivity and sensory functional recovery in rats [24]. Increase of paw withdrawal latency indicates that time to pain perception is increased. Decrease of paw withdrawal latency indicates that time to pain perception is decreased. In our study, paw withdrawal latency was decreased following SNCI, however, dexmedetomidine treatment increased withdrawal latency in SNCI.

Changes in descending inhibitory system and ascending fa-
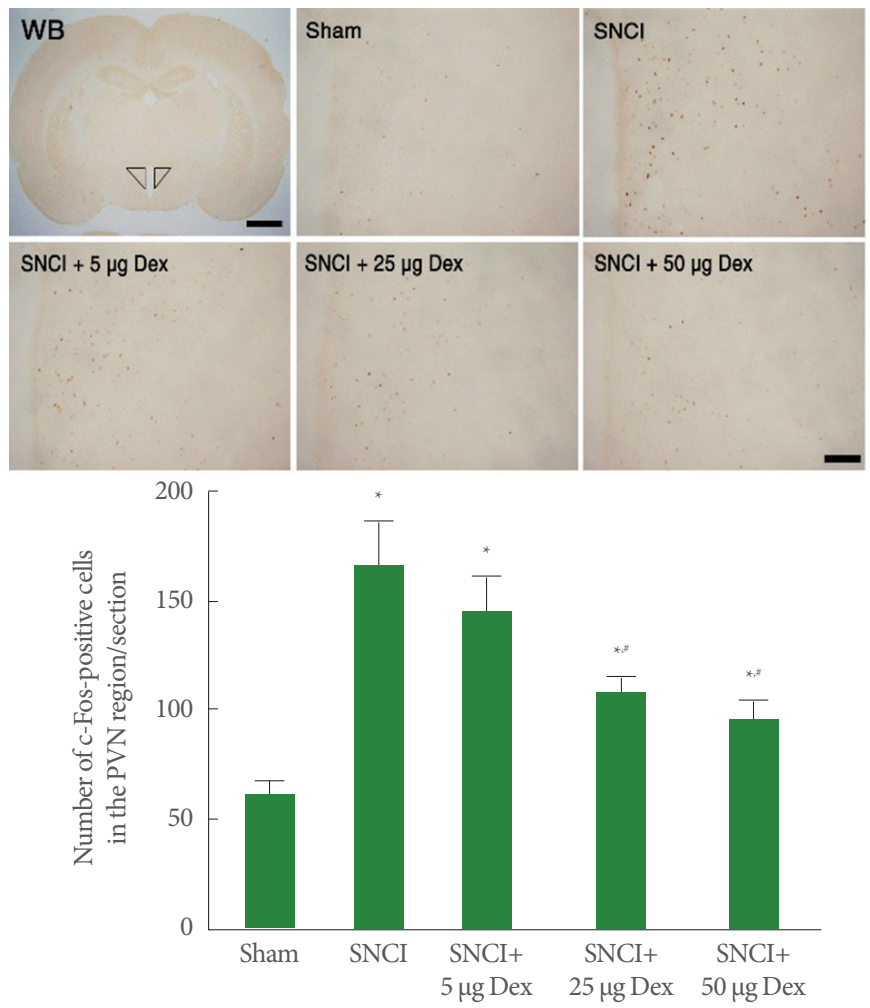
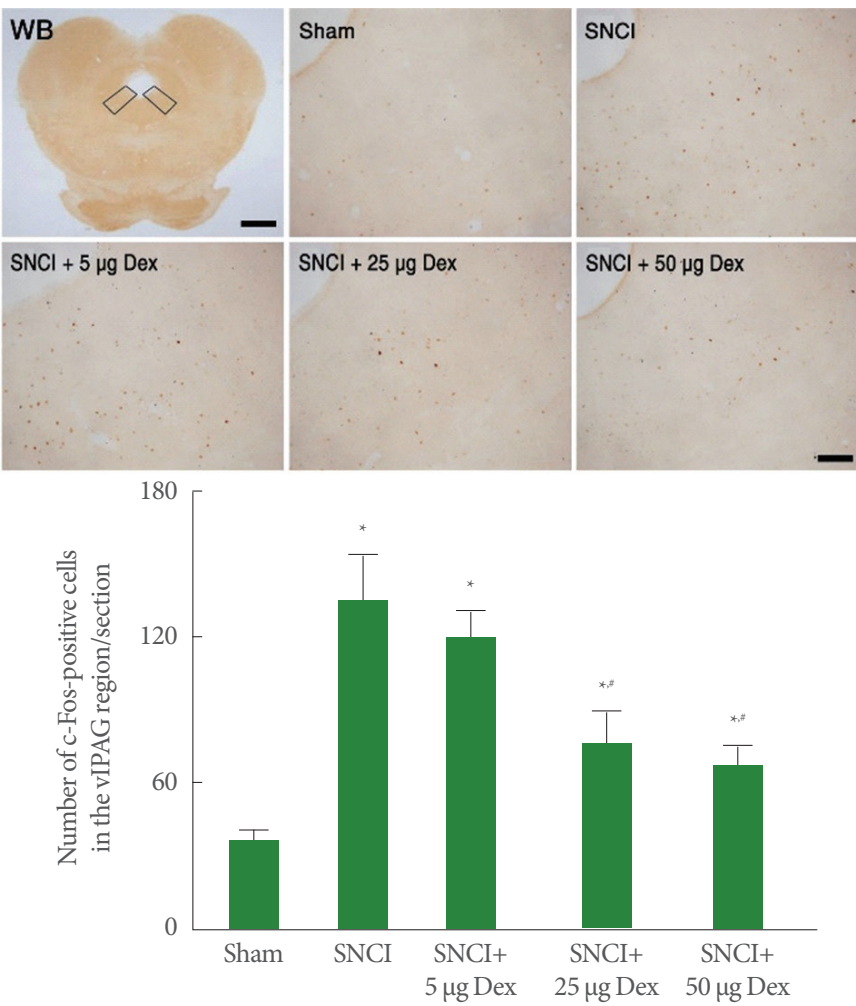

Fig. 3. Effect of dexmedetomidine on c-Fos expressions in the paraventricular nucleus (PVN) and ventrolateral periaqueductal gray (vlPAG) regions. Upper panel: photomicrographs of c-Fos-positive cells in the PVN (left) and vlPAG (right) regions. The scale bars represent $25 \mu \mathrm{m}$ (whole brain, WB) and $150 \mu \mathrm{m}$ (others). $\triangle$, PVN counting areas; $\square$, vlPAG counting areas. Lower panel: number of c-Fos-positive cells in each group. Sham, sham-operated group; SNCI, sciatic nerve crush injury-induced group; SNCI+5 $\mu$ g Dex, SNCI-induced and 5- $\mu \mathrm{g} / \mathrm{kg}$ dexmedetomidine treatment group; SNCI+25 $\mu$ g Dex, SNCI-induced and 25- $\mu \mathrm{g} / \mathrm{kg}$ dexmedetomidine treatment group; SNCI+50 $\mu \mathrm{g}$ Dex, SNCI-induced and $50 \mu \mathrm{g} / \mathrm{kg}$ dexmedetomidine treatment group. ${ }^{\star} \mathrm{P}<0.05$ compared to the shamoperated group. ${ }^{\#} \mathrm{P}<0.05$ compared to the $\mathrm{SNCI}$-induced group. 

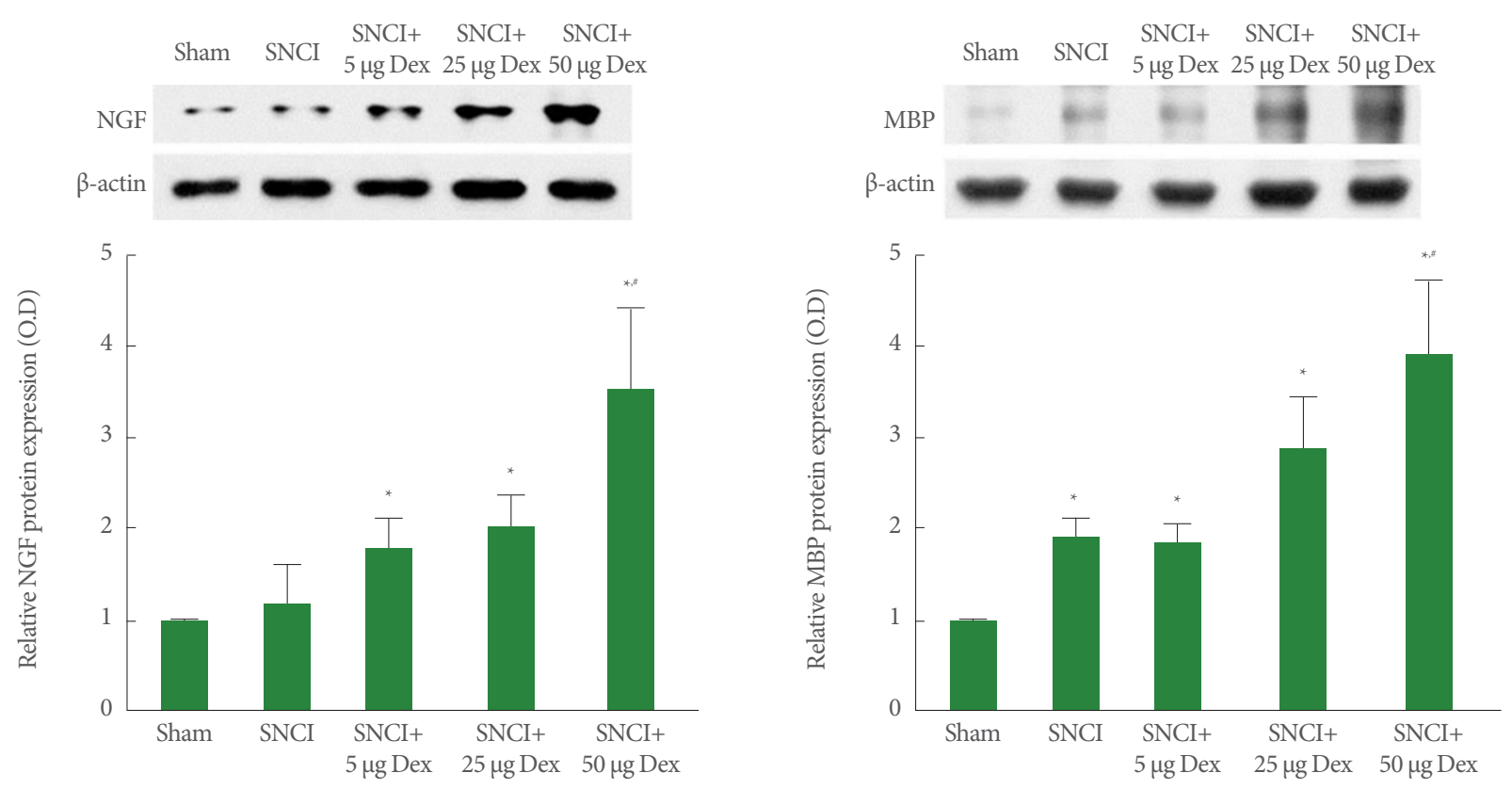

Fig. 4. Effect of dexmedetomidine on nerve growth factor (NGF) and myelin basic protein (MBP) expression in the sciatic nerve. Actin was used as an internal control $(46 \mathrm{kDa})$. Upper panel: the results of band detection using the enhanced chemiluminescence detection kit. Lower panel: The relative expressions of NGF (left) and MBP (right) in each group. Sham, sham-operated group; SNCI, sciatic nerve crush injury-induced group; SNCI+5 $\mu$ g Dex, SNCI-induced and 5- $\mu \mathrm{g} / \mathrm{kg}$ dexmedetomidine treatment group; SNCI+25 $\mu \mathrm{g}$ Dex, SNCI-induced and $25-\mu \mathrm{g} / \mathrm{kg}$ dexmedetomidine treatment group; SNCI+50 $\mu \mathrm{g}$ Dex, SNCI-induced and $50-\mu \mathrm{g} / \mathrm{kg}$ dexmedetomidine treatment group. ${ }^{*} \mathrm{P}<0.05$ compared to the sham-operated group. ${ }^{\sharp} \mathrm{P}<0.05$ compared to the SNCI-induced group.

cilitatory system cause sustained pain [25,26]. Painful experiences have been shown to induce c-Fos expression in the dorsal horn of spinal cord, anterior cingulate cortex, PVN, and PAG of brain $[5,27]$. The frontal cortex, thalamus, and PAG are the main structures for coordination of pain perception. Ligation of sciatic nerve increased the level of c-Fos expression in these brain regions [28]. Increase of c-Fos expression indicates that pain transmitted to the PVN and vlPAG was enhanced. In our study, SNCI increased the number of c-Fos-positive cells in the PVN and vlPAG, indicating that SNCI activated these nociceptive centers.

Activation of the $\alpha_{2}$-adrenoceptor by dexmedetomidine produced analgesia [29]. Higher dosage of dexmedetomidine affected thermal latency and mechanical threshold in both injured and uninjured hindlimbs, demonstrating that dexmedetomidine has antinociceptive and analgesic effects [17]. It is known that the $\alpha_{2}$-adrenoceptor is implicated in reducing allodynia and inhibiting hyperalgesia [30]. In addition, $\alpha_{2}$-adrenoceptor agonist suppressed c-Fos overexpression in the dorsal horn of the spinal cord following chronic constriction injury in rats [31]. In our study, dexmedetomidine suppressed c-Fos overexpression in the
PVN and vlPAG caused by SNCI, resulting in alleviation of thermal hyperalgesia.

Enhancing axonal regeneration and promoting reinnervation are important outcomes when developing therapeutic agents. An important direction for ongoing research is the development of therapeutic strategies that enhance axon regeneration and promote selective target repenetration [32]. NGF and MBP are implicated in promoting nerve repair and enhancing functional recovery after sciatic nerve damage $[9,11]$. NGF expression correlates with hyperalgesia in tissue inflammation [33]. NGF facilitated nerve regeneration and recovery of motor and sensory function after SNCI in rats [13]. NGF expression in the brachial plexus was enhanced at 6 hours after injection of dexmedetomidine [16]. MBP expression is implicated in peripheral nerve regeneration [14]. Close correlation exists between MBP density and functional recovery following sciatic nerve injury [34]. Increase of NGF and MBP indicates promoting nerve repair and enhancing functional recovery. In our study, NGF and MBP expression in sciatic nerve was enhanced by SNCI. In addition, NGF and MBP expression was more potently increased by dexmedetomidine treatment, suggesting that dexmedetomidine 
promoted nerve regeneration in SNCI rats.

Our findings indicate that SNCI caused a deterioration in locomotor function and increased thermal pain sensitivity. However, dexmedetomidine treatment improved locomotor function by upregulating NGF and MBP expressions in the sciatic nerve of SNCI. Dexmedetomidine treatment alleviated thermal hyperalgesia through down-regulation of c-Fos expression in the vlPAG and PVN after SNCI. These effects of dexmedetomidine suggest he possibility that dexmedetomidine is also effective for intractable urogenital ulceration such as urinary bladder ulcers. Dexmedetomidine may be used as a potential new treatment drug for recovery of locomotion and control of pain in peripheral nerve injury.

\section{AUTHOR CONTRIBUTION STATEMENT}

- Conceptualization: $M S J$, JWY

- Formal Analysis: DOK, BJL

- Investigation: JHH, GW, JIK, JYC

- Methodology: JHH, GW, JIK, JYC

- Project Administration: JWY

-Writing-Original Draft: MSJ

· Writing-Review \& Editing: MSJ

\section{REFERENCES}

1. Wang Y, Shen W, Yang L, Zhao H, Gu W, Yuan Y. The protective effects of Achyranthes bidentata polypeptides on rat sciatic nerve crush injury causes modulation of neurotrophic factors. Neurochem Res 2013;38:538-46.

2. Rosberg HE, Carlsson KS, Dahlin LB. Prospective study of patients with injuries to the hand and forearm: costs, function, and general health. Scand J Plast Reconstr Surg Hand Surg 2005;39:360-9.

3. Shin KM, Ko IG, Kim SE, Jin JJ, Hwang L, Kim SH, et al. Low-frequency electroacupncture improves locomotor function after sciatic crushed nerve injury in rats. J Exerc Rehabil 2018;14:927-33.

4. Jensen TS, Gottrup H, Sindrup SH, Bach FW. The clinical picture of neuropathic pain. Eur J Pharmacol 2001;429:1-11.

5. Lee BK, Kim CJ, Shin MS, Cho YS. Diosgenin improves functional recovery from sciatic crushed nerve injury in rats. J Exerc Rehabil 2018;14:566-72.

6. Lee JM, Kim TW, Park HS, Park SS, Shin MS, Sung YH, et al. Effects of exercise on sexual function and central mechanism in the streptozotocin-induced diabetic rats. J Exerc Rehabil 2018;14:10-5.

7. Ko IG, Hwang L, Jin JJ, Kim SH, Han JH, Jeon JW, et al. Add-on therapy with the $a$-blockers tamsulosin and naftopidil improves voiding function by enhancing neuronal activity in prostatic hyperplasia rats. Int Neurourol J 2018;22:20-9.

8. Omura K, Ohbayashi M, Sano M, Omura T, Hasegawa T, Nagano A. The recovery of blood-nerve barrier in crush nerve injury-a quantitative analysis utilizing immunohistochemistry. Brain Res 2004;1001:13-21.

9. Pan HC, Yang DY, Ou YC, Ho SP, Cheng FC, Chen CJ. Neuroprotective effect of atorvastatin in an experimental model of nerve crush injury. Neurosurgery 2010;67:376-89.

10. Tuszynski MH, Thal L, Pay M, Salmon DP, U HS, Bakay R, et al. A phase 1 clinical trial of nerve growth factor gene therapy for Alzheimer disease. Nat Med 2005;11:551-5.

11. Sun W, Sun C, Lin H, Zhao H, Wang J, Ma H, et al. The effect of collagen-binding NGF- $\beta$ on the promotion of sciatic nerve regeneration in a rat sciatic nerve crush injury model. Biomaterials 2009; 30:4649-56.

12. Hanani M. Satellite glial cells in sensory ganglia: from form to function. Brain Res Brain Res Rev 2005;48:457-76.

13. Yu H, Liu J, Ma J, Xiang L. Local delivery of controlled released nerve growth factor promotes sciatic nerve regeneration after crush injury. Neurosci Lett 2014;566:177-81.

14. Yin Q, Kemp GJ, Yu LG, Wagstaff SC, Frostick SP. Expression of Schwann cell-specific proteins and low-molecular-weight neurofilament protein during regeneration of sciatic nerve treated with neurotrophin-4. Neuroscience 2001;105:779-83.

15. Taveggia C, Pizzagalli A, Fagiani E, Messing A, Feltri ML, Wrabetz L. Characterization of a Schwann cell enhancer in the myelin basic protein gene. J Neurochem 2004;91:813-24.

16. Han JH, Kim DO, Yi JW, Park SW, Kang WJ, Choi YK, et al. Dexmedetomidine, $a_{2}$-adrenoceptor agonist, does not induce apoptosis in the brachial plexus of rats. Anim Cells Syst 2014;18:407-15.

17. Xu M, Kontinen VK, Kalso E. Effects of radolmidine, a novel a2adrenergic agonist compared with dexmedetomidine in different pain models in the rat. Anesthesiology 2000;93:473-81.

18. Malmberg AB, Hedley LR, Jasper JR, Hunter JC, Basbaum AI. Contribution of $\alpha_{2}$ receptor subtypes to nerve injury-induced pain and its regulation by dexmedetomidine. Br J Pharmacol 2001;132: 1827-36.

19. Xu B, Zhang WS, Yang JL, Xu H, Deng XM, Zhang YQ. Dexmedetomidine blocks thermal hyperalgesia and spinal glial activation in rat model of monoarthritis. Acta Pharmacol Sin 2010;31:523-30.

20. Hwang L, Choi IY, Kim SE, Ko IG, Shin MS, Kim CJ, et al. Dexmedetomidine ameliorates intracerebral hemorrhage-induced memory impairment by inhibiting apoptosis and enhancing brain-de- 
rived neurotrophic factor expression in the rat hippocampus. Int $\mathrm{J}$ Mol Med 2013;31:1047-56.

21. Ko IG, Kim SE, Hwang L, Jin JJ, Kim CJ, Kim BK, et al. Late starting treadmill exercise improves spatial leaning ability through suppressing CREP/BDNF/TrkB signaling pathway following traumatic brain injury in rats. J Exerc Rehabil 2018;14:327-34.

22. Ko IG, Moon BM, Kim SE, Jin JJ, Hwang L, Ji ES, et al. Effects of combination treatment of alpha 1-adrenergic receptor antagonists on voiding dysfunction: Study on target organs in overactive bladder rats. Int Neurourol J 2016;20:150-8.

23. Kanyadhara S, Dodoala S, Sampathi S, Punuru P, Chinta G. Ethanolic extract of Aloe vera ameliorates sciatic nerve ligation induced neuropathic pain. Anc Sci Life 2014;33:208-15.

24. Beigi MH, Ghasemi-Mobarakeh L, Prabhakaran MP, Karbalaie K, Azadeh H, Ramakrishna S, et al. In vivo integration of poly $(\varepsilon-$ caprolactone)/gelatin nanofibrous nerve guide seeded with teeth derived stem cells for peripheral nerve regeneration. J Biomed Mater Res A 2014;102:4554-67.

25. Vanegas H, Schaible HG. Descending control of persistent pain: inhibitory or facilitatory? Brain Res Brain Res Rev 2004;46:295309.

26. Moon LD, Madani R, Vassalli JD, Bunge MB. Neuronal overexpression of tissue-type plasminogen activator does not enhance sensory axon regeneration or locomotor recovery following dorsal hemisection of adult mouse thoracic spinal cord. J Neurosci Res 2006;84:1245-54.

27. Nishimori T, Ikeda T, Terayama R, Ishida Y, Nakamura T, Otahara
N. Effect of ionotropic glutamate receptor antagonists on Fos-like immunoreactivity in the dorsal horn following transection of the rat sciatic nerve. Brain Res 2002;934:81-6.

28. Shin KM, Ko IG, Kim SE, Jin JJ, Hwang L, Kim SH, et al. Low-frequency electroacupncture improves locomotor function after sciatic crushed nerve injury in rats. J Exerc Rehabil 2018;14:927-33.

29. Wu JR, Chen H, Yao YY, Zhang MM, Jiang K, Zhou B, et al. Local injection to sciatic nerve of dexmedetomidine reduces pain behaviors, SGCs activation, NGF expression and sympathetic sprouting in CCI rats. Brain Res Bull 2017;132:118-28.

30. Farghaly HS, Abd-Ellatief RB, Moftah MZ, Mostafa MG, Khedr $\mathrm{EM}$, Kotb HI. The effects of dexmedetomidine alone and in combination with tramadol or amitriptyline in a neuropathic pain model. Pain Physician 2014;17:187-95.

31. Nakamura T, Ikeda T, Takeda R, Igawa K, Naono-Nakayama R, Sakoda $\mathrm{S}$, et al. The role of spinal serotonin receptor and alpha adrenoceptor on the antiallodynic effects induced by intrathecal milnacipran in chronic constriction injury rats. Eur J Pharmacol 2014; 738:57-65.

32. Navarro X, Vivo M, Valero-Cabre A. Neural plasticity after peripheral nerve injury and regeneration. Prog Neurobiol 2007;82:163201.

33. Skaper SD. Nerve growth factor: a neurokine orchestrating neuroimmune-endocrine functions. Mol Neurobiol 2001;24:183-99.

34. Rustemeyer J, Dicke U. Correlation of three sciatic functional indices with histomorphometric findings in a rat sciatic nerve allograft repair model. Microsurgery 2009;29:560-7. 